\title{
On the Carleman determinants
}

by

ROMAN SIKORSKI (Warszawa)

Fredholm [1] defined the notion of determinants and subdeterminants of all orders for the integral equation

$$
x(s)+\int_{0}^{1} \tau(s, t) x(t) d t=x_{0}(s)
$$

with continuous kernel $\tau(s, t)$. Fredholm's determinant theory was recently generalized (Grothendieck [1-2], Leżański [1-2], Ruston [1-2], Sikorski [1-4]; see also the expository paper by Sikorski [5]) over the case of linear equations

$$
x+T x=x_{0}
$$

in arbitrary Banach spaces $X$, under some hypothesis on the operators $T$.

In the general theory of determinants in Banach spaces $X$, two notions of the subdeterminant of an order $n$ can be introduced: the first one coincides with the algebraic notion of subdeterminants in the case where $X$ is finitely dimensional; the second one coincides with the original Fredholm subdeterminant in the case where $X$ is the space $C$ (for details, see Grothendieck [1] and Sikorski [4-5]).

In the case where $X$ is a Hilbert space, the general determinant theory is applicable to (2) if and only if $T$ is nuclear, i. e. (see e. g. Sikorski [2], Theorem V) if $T=T_{1} T_{2}$, where $T_{1}, T_{2}$ are Hilbert-Schmidt operators. An operator $T$ in a Hilbert space $X$ is said to be a HilbertSchmidt operator if, given any orthogonal system of coordinates in $X$ (i. e. a complete orthonormal set in $X$ ), $T$ is represented by an infinite square matrix $\left(\tau_{i, j}\right)$ such that

$$
\|T\|=\sqrt{\sum_{i, j}\left|\tau_{i, j}\right|^{2}}<\infty .
$$

In the case of the Hilbert space $\boldsymbol{L}^{2}$, Carleman [1] (see also Hille and Tamarkin [1-2], Smithies [2]) defined the notion of determinant and subdeterminants for the integral equation (1) under the hypothesis that

$$
\int_{0}^{1} \int_{0}^{1}|\tau(s, t)|^{2} d s d t<\infty
$$


Carleman's formulae were a simple modification of those of Fredholm: everywhere the expressions $\int_{0}^{1} \tau(s, s) d s$ were replaced by zero, following an idea of Hilbert [1].

In the abstract formulation (2), the case investigated by Carleman is that where $T$ is a Hilbert-Schmidt operator. A very interesting treatment of the Carleman determinant and the first subdeterminant in an abstract Hilbert space $X$ is due to Smithies [1] who also gave a simple proof of the convergence of series defining the determinant and the first subdeterminant $\left({ }^{1}\right)$.

The subject of this paper is to give a complete determinant theory for the equation (2) in an abstract Hilbert space $X, T$ being a HilbertSchmidt operator. The subdeterminants of all orders are defined. Similarly, as in the general determinant theory in Banach spaces, two notions of subdeterminants of an order $n$ are introduced. They are called, respectively, the Carleman subdeterminant and the Carleman-Fredholm subdeterminant. Carleman's original subdeterminants coincide with the Carleman-Fredholm subdeterminants, in the terminology assumed in this paper.

It is easy to know what formulae should define the subdeterminants of (2). Indeed, the subdeterminants should be defined by the same series as in the case of a nuclear $T$, with only one modification: everywhere the trace of $T$ (i. e. the abstract substitute of $\int_{0}^{1} \tau(s, s) d s$ ) should be replaced by 0 . The only difficulty is to prove that the series converge. Fortunately, this difficulty is only on the surface. Using an argument of Grothendieck [1], the convergence of series defining the subdeterminants can be deduced from the Carleman-Smithies theorem stating the convergence of the series defining the determinant.

\$1. Terminology and notation. We shall consider a fixed Hilbert space $X$. The letter $E$ will denote the Hilbert space of all linear bounded functionals on $X$. The letters $x, y, z$ (with indices, if necessary) denote always elements of $X$, and the letters $\xi, \eta, \zeta$ - elements of $\Xi$. The value of a functional $\xi$ at a point $x$ is denoted by $\xi x$.

The symbol $\mathfrak{O}_{n}$ will denote the Banach space of all $2 n$-linear bounded functionals on $\Xi^{n} \times X^{n}$. If $B \in \mathfrak{D}_{n}$, then the value of $B$ at a point $\left(\xi_{1}, \ldots, \xi_{n}, x_{1}, \ldots, x_{n}\right) \in \Xi^{n} \times X^{n}$ will be usually denoted by

$$
B\left(\begin{array}{l}
\xi_{1}, \ldots, \xi_{n} \\
x_{1}, \ldots, x_{n}
\end{array}\right) .
$$

(1) For another theory of determinants of operators in an abstract Hilbert space, see Fuglede and Kadison $[1,2]$.
The norm of $B$ in $\mathfrak{O}_{n}$ is

$$
|B|=\sup _{\left|\xi_{1}\right| \leqslant 1, \ldots,\left|\xi_{n}\right| \leqslant 1,\left|x_{1}\right| \leqslant 1, \ldots,\left|x_{n}\right| \leqslant 1} \mid B\left(\begin{array}{c}
\xi_{1}, \ldots, \xi_{n} \\
a_{1}, \ldots, x_{n}
\end{array}\right) .
$$

In particular, $\mathfrak{O}_{1}$ is the Banach space of all bounded bilinear functionals $A$ on $\Xi \times X$. If $A \in \mathfrak{O}_{1}$, we write also $\xi A x$ instead of $A\left(\begin{array}{l}\xi \\ x\end{array}\right)$. Ele-
ments in $\mathfrak{O}_{1}$ are called operators.

Let $A \in \mathfrak{O}_{1}$. For every $x$ there exists exactly one $y$ such that $\xi y=\xi A x$ for every $\xi$. We denote this element $y$ by $A x$. Similarly, for every $\xi$ there exists exactly one $\eta$ such that $\eta x=\eta A x$ for every $x$. We denote this element $\eta$ by $\xi A$. Obviously, the mappings $y=A x$ and $\eta=\xi A$ are adjoint endomorphisms in $X$ and $\Xi$ respectively, and their ordinary norms coincide with the norm $|A|$ of $A$ in $\mathfrak{O}_{1}$. Conversely, every endomorphism in $X$ or in $E$ is of the above form. Thus every operator can be simultaneously interpreted as a bilinear functional on $\Xi \times X$, or an endomorphism in $X$, or as an endomorphism in $\Xi$. The three interpretations of any $A \in \mathfrak{D}_{1}$ will be systematically used in this paper.

The set $\mathfrak{D}_{1}$ of operators is a Banach algebra with the following definition of the product $A_{1} A_{2}$ of $A_{1}, A_{2} \in \mathfrak{O}_{1}$ :

$$
\xi\left(A_{1} A_{2}\right) x=\left(\varrho A_{1}\right)\left(A_{2} x\right) .
$$

In other words, the product $A_{1} A_{2}$ interpreted as an endomorphism in $X$ (in $\Xi$ ) is the superposition of the endomorphism $A_{1}, A_{2}$ in $X$ (of the endomorphism $A_{2}, A_{1}$ in $\Xi$ ). The unit element of the algebra $\mathfrak{O}_{1}$ is the fundamental bilinear functional $I$ :

$$
\xi I x=\xi x .
$$

By definition, $I x=x$ and $\xi I=\xi$ for all $x$ and $\xi$.

Let $x_{0}, \xi_{0}$ be fixed. The operator $K_{0}$ defined by the formula

$$
\xi K_{0} x=\xi x_{0} \cdot \xi_{0} x
$$

(i. e. the product of numbers $\varrho x_{0}$ and $\xi_{0} x$ ) is called one-dimensional and denoted by $x_{0} \cdot \xi_{0}$. By definition, $K_{0} x=x_{0} \cdot \xi_{0} x_{1}$ and $\xi K=\xi x_{0} \cdot \xi_{0}$ (the dot replaces here parentheses).

Any finite sum $K=\sum_{i=1}^{m} x_{i} \cdot \xi_{i}$ of one-dimensional operators is called finitely dimensional operator.

The letter $T$ denotes always Hilbert-Schmidt operators, and the letter $\mathfrak{S}$ denotes the Banach algebra of all Hilbert-Schmidt operators $T$ with the norm $\|T\|$ defined by (3). $\mathfrak{S}$ is an ideal in $\mathfrak{D}_{1}$. Moreover,

$$
\left\|x_{0} \cdot \xi_{0}\right\|=\left|x_{0}\right| \cdot\left|\xi_{0}\right|
$$

$|T| \leqslant\|T\|$ for every $T \in \mathcal{S}$, 
and

$\left(4^{\prime \prime}\right) \quad\|T A\| \leqslant\|T\| \cdot|A|,\|A T\| \leqslant|A| \cdot\|T\|$ for every $A \in \mathcal{O}_{1}$ and $T \epsilon \Im$ .

Any nuclear operator $T$, i. e. a product $T=T_{1} T_{2}$ of two Schmidt operators $T_{1}, T_{2}$, has a well defined trace denoted by $\operatorname{tr} T$. Viz. if, in a given system of orthogonal coordinates in $X, T_{1}$ and $T_{2}$ are represented by matrices $\left(\tau_{i, j}\right)$ and $\left(\sigma_{i, j}\right)$ respectively, then

$$
\operatorname{tr} T=\sum_{i, j} \tau_{j, i} \sigma_{i, j}
$$

The number $\operatorname{tr} T$ just defined depends neither on the representation of $T$ in the form $T_{1} T_{2}$, nor on the choice of the system of coordinates in $X$. We have

$$
\operatorname{tr} S T=\operatorname{tr} T S \quad \text { and } \quad|\operatorname{tr} S T| \leqslant\|S\| \cdot\|T\|
$$

for $T, S \in \mathcal{G}$.

Suppose that $B\left(\begin{array}{l}\xi_{1}, \ldots, \xi_{n} \\ x_{1}, \ldots, x_{n}\end{array}\right)$ is a $2 n$-linear functional on $\Xi^{n} \times X^{n}$ such that $B$ considered as function of $\xi_{n}$ and $x_{n}$ only is a Hilbert-Schmidt operator, i. e., for any fixed $\xi_{1}, \ldots, \xi_{n-1}, x_{1}, \ldots, x_{n-1}$,

$$
B\left(\begin{array}{l}
\xi_{1}, \ldots, \xi_{n} \\
x_{1}, \ldots, x_{n}
\end{array}\right)=\xi_{n} S x_{n},
$$

where $S \in \mathcal{S}$. Let $T \in \mathcal{G}$. The number $\operatorname{tr} T S$ will also be denoted by

$$
T_{\xi_{n} x_{n}} B\left(\begin{array}{l}
\xi_{1}, \ldots, \xi_{n} \\
x_{1}, \ldots, x_{n}
\end{array}\right) .
$$

The number (6) does not depend on the bound variables $\xi_{n}, x_{n}$, but it depends on $\xi_{1}, \ldots, \xi_{n-1}, x_{1}, \ldots, x_{n-1}$, viz. it is a $(2 n-2)$-linear functional $B_{1}\left(\begin{array}{l}\xi_{1}, \ldots, \xi_{n-1} \\ x_{1}, \ldots, x_{n-1}\end{array}\right)$ on $\Xi^{n-1} \times X^{n-1}$. Suppose that $B_{1}$ interpreted as a function of $\xi_{n-1}$ and $x_{n-1}$ only is a Hilbert-Schmidt operator, and that $T^{\prime} \in \mathfrak{S}$. Then

$$
T_{\xi_{n-1} x_{n-1}}^{\prime} T_{\xi_{n} x_{n}} B\left(\begin{array}{l}
\xi_{1}, \ldots, \xi_{n} \\
x_{1}, \ldots, x_{n}
\end{array}\right)
$$

is the number $T_{\xi_{n-1} x_{n-1}}^{\prime} B_{1}\left(\begin{array}{l}\xi_{1}, \ldots, \xi_{n-1} \\ x_{1}, \ldots, x_{n-1}\end{array}\right)$. Continuing this procedure we can define, under similar hypotheses, the expression

$$
T_{r \xi_{r} x_{r} \ldots T_{1 \xi_{1} x_{1}} B}\left(\begin{array}{l}
\xi_{1}, \ldots, \xi_{n} \\
x_{1}, \ldots, x_{n}
\end{array}\right) \quad(r \leqslant n)
$$

for $T_{1}, \ldots, T_{r} \in \mathfrak{S}$. It is not difficult to verify that the required hypotheses are fulfilled in the case where

$$
B\left(\begin{array}{l}
\xi_{1}, \ldots, \xi_{n} \\
x_{1}, \ldots, x_{n}
\end{array}\right)=\left|\begin{array}{ccccc}
0 & \xi_{1} x_{2} & \xi_{1} x_{3} & \ldots & \xi_{1} x_{n} \\
\xi_{2} x_{1} & 0 & \xi_{2} x_{3} & \ldots & \xi_{2} x_{n} \\
\xi_{3} x_{1} & \xi_{3} x_{2} & 0 & \ldots & \xi_{3} x_{n} \\
\ldots & \ldots & \ldots & \ldots & \ldots \\
\xi_{n} x_{1} & \xi_{n} x_{2} & \xi_{n} x_{3} & \ldots & 0
\end{array}\right| .
$$

In the sequel we shall consider some analytic functions $D(T)$ on $\subseteq$ and some analytic mappings $D(T)$ from $\mathcal{G}$ into a Banach space. Then $D^{\prime}\left(T ; T_{1}\right)$ will denote the first differential of $D(T)$, i. e.

By induction,

$$
D^{\prime}\left(T ; T_{1}\right)=\lim _{\varepsilon \rightarrow 0} \varepsilon^{-1}\left(D\left(T+\varepsilon T_{1}\right)-D(T)\right)
$$

$$
\begin{aligned}
& D^{(m)}\left(T ; T_{1}, \ldots, T_{m}\right) \\
& \quad=\lim _{\varepsilon \rightarrow 0} \varepsilon^{-1}\left(D\left(T+\varepsilon T_{m} ; T_{1}, \ldots, T_{m-1}\right)-D\left(T ; T_{1}, \ldots, T_{m-1}\right)\right)
\end{aligned}
$$

for $T, T_{1}, \ldots, T_{m} \in \mathfrak{S}$. Clearly $D^{(m)}\left(T ; T_{1}, \ldots, T_{m}\right)$ is linear and symmetric in variables $T_{1}, \ldots, T_{m}$.

If values of $D(T)$ belong to $\mathfrak{O}_{n}$, then also $D^{(m)}\left(T ; T_{1}, \ldots, T_{m}\right) \in \mathfrak{O}_{n}$, and $D^{(m)}\left(T ; T_{1}, \ldots, T_{m}\right)\left(\begin{array}{l}\xi_{1}, \ldots, \xi_{n} \\ x_{1}, \ldots, x_{n}\end{array}\right)$ is the value of the $2 n$-linear functional $D^{(m)}\left(T ; T_{1}, \ldots, T_{m}\right)$ at the point $\left(\xi_{1}, \ldots, \xi_{n}, x_{1}, \ldots, x_{n}\right) \in \Xi^{n} \times X^{n}$, according to the notation assumed at the beginning of this section. The symbol $D(T)\left(\begin{array}{l}\xi_{1}, \ldots, \xi_{n} \\ x_{1}, \ldots, x_{n}\end{array}\right)$ has the analogous meaning.

§ 2. The Carleman determinant. Let $T \in \mathcal{O}$. By the Carleman determinant of the operator $A=I+T$ we understand the number

$$
D_{0}(T)=\sum_{m=0}^{\infty} \frac{1}{m !} D_{0, m}(T),
$$

where $D_{0,0}(T)=1$ and, for $m>0$,

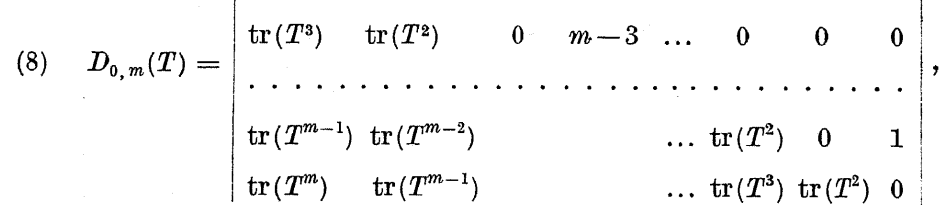


i. e., by an easy verification,

$$
D_{0, m}(T) \stackrel{\prime}{=} T_{\xi_{1} x_{1}} \ldots T_{\xi_{m} x_{m}}\left|\begin{array}{ccccc}
0 & \xi_{1} x_{2} & \xi_{1} x_{3} & \ldots & \xi_{1} x_{m} \\
\xi_{2} x_{1} & 0 & \xi_{2} x_{3} & \ldots & \xi_{2} x_{m} \\
\xi_{3} x_{1} & \xi_{3} x_{2} & 0 & \ldots & \xi_{3} x_{m} \\
\ldots & \ldots & \ldots & \ldots & \ldots \\
\xi_{m} x_{1} & \xi_{m} x_{2} & \xi_{m} x_{3} & \ldots & 0
\end{array}\right|
$$

Carleman [1] and Smithies [1] have proved that

$$
\left|D_{0, m}(T)\right| \leqslant m !\left(\frac{e}{m}\right)^{m / 2}\|T\|^{m}
$$

Thus the series (7) converges absolutely. It follows from (9) that $D_{0, m}(T)$ is a homogeneous polynomial of $T$ of the degree $m$. Hence it follows that $D_{0}(T)$ is an analytic function defined on the Banach space $\mathbb{G}$. Carleman [1] and Smithies [1] have proved that

$$
\left|D_{0}(T)\right| \leqslant \exp \left(\frac{1}{2}\|T\|^{2}\right) .
$$

By the general theory of homogeneous polynomials (see e. g. Hille and Phillips [1], Chapter XXVI), there exists an $m$-linear functional $\theta_{m}\left(T_{1}, \ldots, T_{m}\right)$ on $\mathfrak{S}^{m}$, symmetric in variables $T_{1}, \ldots, T_{m} \in \mathfrak{S}$, such that

$$
D_{0, m}(T)=\theta_{m}(T, \ldots, T) \text {. }
$$

The functional $\theta_{m}$ is uniquely determined by $D_{0, m}$, viz.

$$
\theta_{m}\left(T_{1}, \ldots, T_{m}\right)=\frac{1}{m !} \sum(-1)^{i_{1}+\ldots+i_{m}+m} D_{0, m}\left(i_{1} T_{1}+\ldots+i_{m} T_{m}\right)
$$

where the summation is extended over all sequences $i_{1}, \ldots, i_{m}$ composed of numbers 0 and 1 . It follows easily from (10) and (13) that the norm

$$
\left|\theta_{m}\right|=\sup _{\left\|T_{1}\right\| \leqslant 1, \ldots,\left\|T_{m}\right\| \leqslant 1}\left|\theta_{m}\left(T_{1}, \ldots, T_{m}\right)\right|
$$

of $\theta_{m}$ satisfies the inequality

$$
\left|\theta_{m}\right| \leqslant m^{m / 2}(2 \sqrt{e})^{m}
$$

We can write an explicit formula for $\theta_{m}$ :

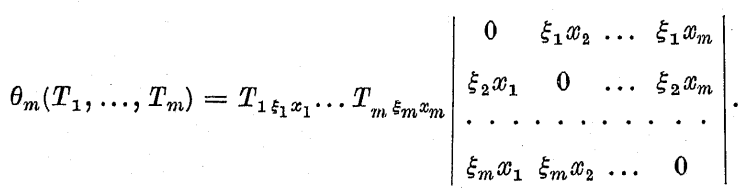

In fact, the right side of (15) is a symmetric functional satisfying (12) on account of (9). The detailed proof of the symmetry is similar to an argument in Leżański [1], p. 248.

Since

$$
D_{0, m}^{(n)}\left(T ; T_{1}, \ldots, T_{n}\right)= \begin{cases}\frac{m !}{(m-n) !} \theta_{m}\left(T, \ldots, T, T_{1}, \ldots, T_{n}\right) & \text { for } n \leqslant m, \\ 0 & \text { for } n>m,\end{cases}
$$

we have

$$
D^{(n)}\left(T ; T_{1}, \ldots, T_{n}\right)=\sum_{m=0}^{\infty} \frac{1}{m !} \theta_{m+n}\left(T, \ldots, T, T_{1}, \ldots, T_{n}\right)
$$

By (14),

$$
\begin{aligned}
\mid \theta_{m+n}\left(T, \ldots, T, T_{1}, \ldots,\right. & \left.T_{n}\right) \mid \\
& \leqslant(n+m)^{\frac{n+m}{2}}(2 \sqrt{e})^{n+m}\|T\|^{m}\left\|T_{1}\right\| \ldots\left\|T_{n}\right\| .
\end{aligned}
$$

Putting $T_{1}=x_{1} \cdot \xi_{1}, \ldots, T_{n}=x_{n} \cdot \xi_{n}$ we obtain (see (4))

$$
\begin{aligned}
\mid \theta_{m+n}(T, \ldots, & \left.T, x_{1} \cdot \xi_{1}, \ldots, x_{n} \cdot \xi_{n}\right) \mid \\
& \leqslant(n+m)^{\frac{n+m}{2}}(2 \sqrt{e})^{n+m}\|T\|^{m}\left|x_{1}\right| \cdot\left|\xi_{1}\right| \cdot \ldots \cdot\left|x_{n}\right| \cdot\left|\xi_{n}\right| .
\end{aligned}
$$

For every fixed $T \epsilon \mathcal{S}$ the expressions $\theta_{m+n}\left(T, \ldots, T, x_{1} \cdot \xi_{1}, \ldots, x_{n} \cdot \xi_{n}\right)$ and (18) $\quad D_{0}^{(n)}\left(T ; x_{1} \cdot \xi_{1}, \ldots, x_{n} \cdot \xi_{n}\right)$

$$
=\sum_{m=0}^{\infty} \frac{1}{m !} \theta_{m+n}\left(T, \ldots, T, x_{1} \cdot \xi_{1}, \ldots, x_{n} \cdot \xi_{n}\right)
$$

(see (16)) are $2 n$-linear bounded functionals on $\Xi^{n} \times X^{n}$, i. e. element of $\mathfrak{O}_{n}$. It follows from (17) that, for every fixed $T \epsilon \mathfrak{S}$, the series of element of $\mathfrak{O}_{n}$ on the right side of (18) converges in norm to the left side of (18).

\$ 3. The Carleman subdeterminants. By the first Carleman subdeterminant of an operator $A=I+T(T \in \mathcal{S})$ we shall understand the bilinear functional $D_{1}(T)$ defined by the equality

$$
\xi D_{1}(T) x=D_{1}(T)\left(\begin{array}{l}
\xi \\
x
\end{array}\right)=D_{0}^{\prime}(T ; x \cdot \xi)+D_{0}(T) \cdot \xi x .
$$

By induction, the $n$-th Carleman subdeterminant of $A=I+T$ is the $2 n$-linear functional $D_{n}(T)$ defined by the equality

$$
\begin{aligned}
(20) \quad & D_{n}(T)\left(\begin{array}{l}
\xi_{1}, \ldots, \xi_{n} \\
x_{1}, \ldots, x_{n}
\end{array}\right) \\
& =D_{n-1}^{\prime}\left(T ; x_{n} \cdot \xi_{n}\right)\left(\begin{array}{l}
\xi_{1}, \ldots, \xi_{n-1} \\
x_{1}, \ldots, x_{n-1}
\end{array}\right)+D_{n-1}(T)\left(\begin{array}{l}
\xi_{1}, \ldots, \xi_{n-1} \\
x_{1}, \ldots, x_{n-1}
\end{array}\right) \cdot \xi_{n} x_{n} .
\end{aligned}
$$


The differentiation on the right side is feasible because one proves, by induction, that $D_{n}(T)$ is an analytic mapping from $\mathfrak{\subseteq}$ into $\mathfrak{D}_{n}$. Consequently

$$
D_{n}(T)=\sum_{m=0}^{\infty} \frac{1}{m !} D_{n, m}(T)
$$

where $D_{n, m}(T)$ is a homogeneous polynomial (of the variable $T \in \mathfrak{S}$ ) of the degree $m$, with values in $\mathfrak{O}_{n}$. It is not difficult to prove by induction on $n$ that

(22) $\quad D_{n, m}(T)\left(\begin{array}{l}\xi_{1}, \ldots, \xi_{n} \\ x_{1}, \ldots, x_{n}\end{array}\right)$

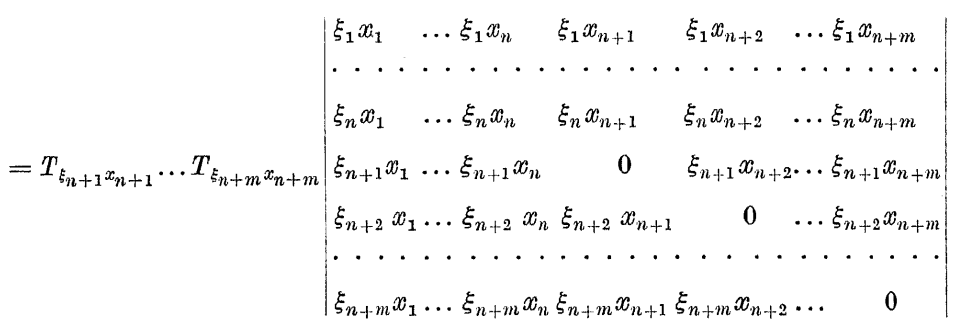

Hence it follows that

$$
D_{n, m}(T)=\left|\begin{array}{cccccccc}
T_{n}^{0}, & m, & 0, & 0, & 0, & \ldots, & 0, & 0 \\
T_{n}^{1}, & 0, & m-1, & 0, & 0, & \ldots, & 0, & 0 \\
T_{n}^{2}, & \operatorname{tr} T^{2}, & 0, & m-2, & 0, & \ldots, & 0, & 0 \\
T_{n}^{3}, & \operatorname{tr} T^{3}, & \operatorname{tr} T^{2}, & 0, & m-3, \ldots, & 0, & 0 \\
\ldots & \ldots & \ldots & \ldots & \ldots & \ldots & \ldots & \ldots \\
T_{n}^{m-1}, & \operatorname{tr} T^{m-1}, & \operatorname{tr} T^{m-2}, & & \ldots, & 0, & 1 \\
T_{n}^{m}, & \operatorname{tr} T^{m}, & \operatorname{tr} T^{m-1}, & \ldots, & \operatorname{tr} T^{2}, & 0
\end{array}\right|
$$

where

$$
\begin{aligned}
& \text { (24) } \quad T_{n}^{m}\left(\begin{array}{l}
\xi_{1}, \ldots, \xi_{n} \\
x_{1}, \ldots, x_{n}
\end{array}\right) \\
& =\sum\left|\begin{array}{l}
\xi_{1} T^{i_{1}} x_{1}, \ldots, \xi_{1} T^{i_{1}} x_{1} \\
\ldots \ldots . . . . \\
\xi_{n} T^{i_{n}} x_{1}, \ldots, \xi_{n} T^{i_{n} x_{1}}
\end{array}\right|=\sum\left|\begin{array}{l}
\xi_{1} T^{i_{1}} x_{1}, \ldots, \xi_{1} T^{i_{n}} x_{n} \\
\ldots \ldots . . . . . \\
\xi_{1} T^{i_{1}} x_{1}, \ldots, \xi_{1} T^{i_{n}} x_{n}
\end{array}\right|
\end{aligned}
$$

the summation being extended over all sequences $i_{1}, \ldots, i_{n}$ of non-negative integers whose sum is equal to $m$.
It follows from (22) or (23) that $D_{n, m}(T)\left(\begin{array}{l}\xi_{1}, \ldots, \xi_{n} \\ x_{1}, \ldots, x_{n}\end{array}\right)$ is skew symmetric in $\xi_{1}, \ldots, \xi_{n}$ and in $x_{1}, \ldots, x_{n}$. Thus, by $(21), D_{n}(T)\left(\begin{array}{l}\xi_{1}, \ldots, \xi_{n} \\ x_{1}, \ldots, x_{n}\end{array}\right)$ is also skew symmetric in $\xi_{1}, \ldots, \xi_{n}$ and in $x_{1}, \ldots, x_{n}$.

We can write immediate formulae for $D_{n}(T)$ :

$$
D_{n}(T)\left(\begin{array}{l}
\xi_{1}, \ldots, \xi_{n} \\
x_{1}, \ldots, x_{n}
\end{array}\right)=D_{0}^{(n)}\left(T ; x_{1} \cdot \xi_{1}, \ldots, x_{n} \cdot \xi_{n}\right)+
$$$$
+\sum_{i=1}^{n} D_{0}^{(n-1)}\left(T ; x_{1} \cdot \xi_{1}, \ldots, x_{i-1} \cdot \xi_{i-1}, x_{i+1} \cdot \xi_{i+1}, \ldots, x_{n} \cdot \xi_{n}\right) \cdot \xi_{i} x_{i}+
$$

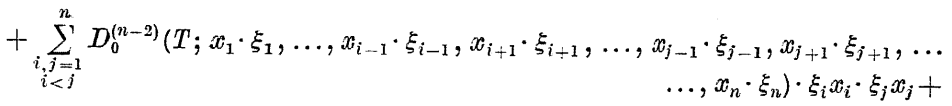
$+\ldots \ldots \ldots \ldots \ldots \ldots \ldots \ldots \ldots$ $+\sum_{i=1}^{n} D_{0}^{\prime}\left(T ; x_{i} \cdot \xi_{i}\right) \cdot \xi_{1} x_{1} \cdot \ldots \cdot \xi_{i-1} x_{i-1} \cdot \xi_{i+1} x_{i+1} \cdot \ldots \cdot \xi_{n} x_{n}+$ $+D_{0}(T) \cdot \xi_{1} x_{1} \cdot \ldots \cdot \xi_{n} x_{n}$.

It follows from (18), (21) and (25) that

$$
\begin{aligned}
& \text { (26) } \quad D_{n, m}(T)\left(\begin{array}{c}
\xi_{1}, \ldots, \xi_{n} \\
x_{1}, \ldots, x_{n}
\end{array}\right)=\theta_{m+n}\left(T, \ldots, T, x_{1} \cdot \xi_{1}, \ldots, x_{n} \cdot \xi_{n}\right)+ \\
& 7 \sum_{i=1}^{n} \theta_{m+n-1}\left(T, \ldots, T, x_{1} \cdot \xi_{1}, \ldots, x_{i-1} \cdot \xi_{i-1}, x_{i+1} \cdot \xi_{i+1}, \ldots, x_{n} \cdot \xi_{n}\right) \cdot \xi_{i} x_{i}+
\end{aligned}
$$

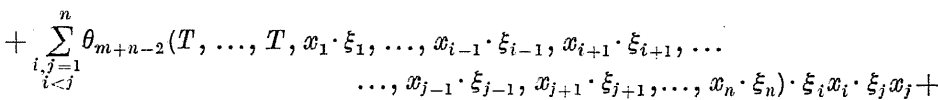

$$
\begin{aligned}
& +\ldots \ldots \ldots \ldots \ldots \ldots \ldots \ldots \ldots \ldots \\
& +\sum_{i=1}^{n} \theta_{m+1}\left(T, \ldots, T, x_{i} \cdot \xi_{i}\right) \cdot \xi_{1} x_{1} \cdot \ldots \cdot \xi_{i-1} x_{i-1} \cdot \xi_{i+1} x_{i+1} \cdot \ldots \cdot \xi_{n} x_{n}+ \\
& +\theta_{m}(T, \ldots, T) \cdot \xi_{1} x_{1} \cdot \ldots \cdot \xi_{n} x_{n} \text {. }
\end{aligned}
$$

By (17), and (26) we have the following estimation of the norm of the 2n-linear functional $D_{n, m}(T)$ :

$$
\left|D_{n, m}(T)\right| \leqslant 2^{n}(n+m)^{\frac{n+m}{2}}\left(2 V^{\prime} e\right)^{n+m}\|T\|^{m} .
$$

This proves that, for every fixed $T \epsilon \mathcal{G}$, the series (21) on elements of $\mathfrak{O}_{n}$ converges in norm. 
Formula (25) expresses $D_{n}(T)$ by means of $D_{0}(T)$ and its derivatives. The following formula expresses the derivatives of $D_{0}(T)$ by means of $D_{0}, \ldots, D_{n}, \ldots$ :

$$
\text { (28) } \begin{aligned}
& D_{0}^{(n)}\left(T ; x_{1} \cdot \xi_{1}, \ldots, x_{n} \cdot \xi_{n}\right)=D_{n}(T)\left(\begin{array}{l}
\xi_{1}, \ldots, x_{n} \\
x_{1}, \ldots, x_{n}
\end{array}\right)- \\
& -\sum_{i=1}^{n} D_{n-1}(T)\left(\begin{array}{l}
\xi_{1}, \ldots, \xi_{i-1}, \xi_{i+1}, \ldots, \xi_{n} \\
x_{1}, \ldots, x_{i-1}, x_{i+1}, \ldots, x_{n}
\end{array}\right) \cdot \xi_{i} x_{i}+ \\
+ & \sum_{i, j=1}^{n} D_{n-2}(T)\left(\begin{array}{l}
\xi_{1}, \ldots, \xi_{i-1}, \xi_{i+1}, \ldots, \xi_{j-1}, \xi_{j+1}, \ldots, \xi_{n} \\
x_{1}, \ldots, x_{i-1}, x_{i+1}, \ldots, x_{j-1}, x_{j+1}, \ldots, x_{n}
\end{array}\right) \cdot \xi_{i} x_{i} \cdot \xi_{j} x_{j}- \\
& -\ldots \ldots \ldots+\ldots \ldots \xi_{n} x_{n}+ \\
+ & (-1)^{n-1} \sum_{i=1}^{n} D_{1}(T)\left(\begin{array}{l}
\xi_{i} \\
x_{i}
\end{array}\right) \cdot \xi_{1} x_{1} \cdot \ldots \cdot \xi_{i-1} x_{i-1} \cdot \xi_{i+1} x_{i+1} \cdot \ldots \\
+ & (-1)^{n} D_{0}(T) \cdot \xi_{1} x_{1} \cdot \ldots \cdot \xi_{n} x_{n} .
\end{aligned}
$$

Formula (28) follows immediately from (20) and (19).

\section{§ 4. Applications to the theory of linear equations. First we} shall prove that

(i) For every Hilbert-Schmidt operator $T$, the sequenoe

$$
D_{0}(T), D_{1}(T), D_{2}(T), \ldots
$$

is a determinant system for the operator $A=I+T$.

For the definition of determinant system-see Sikorski [3] p. 172, conditions $\left(d_{1}\right)-\left(d_{5}\right)$. We have observed in $\S 3$ that $\left(d_{1}\right)$ and $\left(d_{2}\right)$ hold. $\left(d_{3}\right)$ also holds because $X, \Xi$ are Hilbert spaces.

We have to prove $\left(d_{4}\right)$, i. e. that, for every fixed $T \epsilon \in$ , at least one of the multilinear functionals (29) does not vanish identically. Suppose the

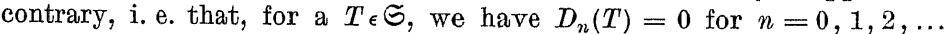
Hence, by (28)

$$
D_{0}^{(n)}\left(T ; T_{1}, \ldots, T_{n}\right)=0
$$

for $n=0,1,2, \ldots$ and for all one-dimensional operators $T_{1}, \ldots, T_{n}$ Since $D_{0}^{(n)}\left(T ; T_{1}, \ldots, T_{n}\right)$ is linear in $T_{1}, \ldots, T_{n},(30)$ holds for all finitely dimensional operators $T_{1}, \ldots, T_{n}$. Since $D_{0}^{(n)}\left(T ; T_{1}, \ldots, T_{n}\right)$ is continuous in $T_{1}, \ldots, T_{n} \in \mathfrak{S}$ and finitely dimensional operators are dense in $\mathfrak{S}$, (30) holds for every $n$ and for all $T_{1}, T_{2}, \ldots, T_{n} \in \mathcal{S}$. Since the analytic function $D_{0}(T)$ vanishes at the point $T$ with all its derivatives, it is equal to zero everywhere. This is impossible since $D_{0}(0)=1$.
Now we have to prove $\left(d_{5}\right)$, i. e. the identities

$$
\begin{aligned}
& D_{n+1}(T)\left(\begin{array}{c}
\xi_{0} A, \xi_{1}, \ldots, \xi_{n} \\
x_{0}, x_{1}, \ldots, x_{n}
\end{array}\right) \\
& \quad=\sum_{i=0}^{n}(-1)^{i} \xi_{0} x_{i} \cdot D_{n}(T)\left(\begin{array}{l}
\xi_{0}, \ldots \ldots . . . ., \xi_{n} \\
x_{0}, \ldots, x_{i-1}, x_{i+1}, \ldots, x_{n}
\end{array}\right), \\
& D_{n+1}(T)\left(\begin{array}{c}
\xi_{0}, \xi_{1}, \ldots, \xi_{n} \\
A x_{0}, x_{1}, \ldots, x_{n}
\end{array}\right) \\
& \quad=\sum_{i=0}^{n}(-1)^{i} \xi_{i} x_{0} \cdot D_{n}(T)\left(\begin{array}{l}
\xi_{0}, \ldots, \xi_{i-1}, \xi_{i+1}, \ldots, \xi_{n} \\
x_{1}, \ldots \ldots . . . \ldots, x_{n}
\end{array}\right) .
\end{aligned}
$$

By the same argument as in Leżański [1], p. 254-256, we prove first those identities for $D_{n+1, m}(T), D_{n, m}(T)$ instead of $D_{n+1}(T), D_{n}(T)$ respectively. Hence we obtain (31), (32).

The next theorem follows immediately from (i) and Sikorski [3], Theorem II.

(ii) Let $T$ be a Hilbert-Schmidt operator, and let $r$ be the smallest integer such that the $2 r$-linear functional $D_{r}$ does not vanish identically. Let $\eta_{1}, \ldots, \eta_{r}$, $y_{1}, \ldots, y_{r}$ be such that

$$
D_{r}(T)\left(\begin{array}{l}
\eta_{1}, \ldots, \eta_{r} \\
y_{1}, \ldots, y_{r}
\end{array}\right) \neq 0
$$

Then there exist elements $\zeta_{1}, \ldots, \zeta_{r}, z_{1}, \ldots, z_{r}$, and an operator $B$ such that, for all $\xi, x$

$$
\begin{aligned}
\xi_{i} x=\frac{D_{r}(T)\left(\begin{array}{l}
\eta_{1}, \ldots \ldots, \ldots, \ldots, \eta_{r} \\
y_{1}, \ldots, y_{i-1}, x, y_{i+1}, \ldots, y_{r}
\end{array}\right)}{D_{r}(T)\left(\begin{array}{l}
\eta_{1}, \ldots, \eta_{r} \\
y_{1}, \ldots, y_{r}
\end{array}\right)} \\
\xi z_{i}=\frac{D_{r}(T)\left(\begin{array}{l}
\eta_{1}, \ldots, \eta_{i-1}, \xi, \eta_{i+1}, \ldots, \eta_{r} \\
y_{1}, \ldots \ldots, \ldots \ldots, \ldots, y_{r}
\end{array}\right)}{D_{r}(T)\left(\begin{array}{l}
\eta_{1}, \ldots, \eta_{r} \\
y_{1}, \ldots, y_{r}
\end{array}\right)} \\
\xi B x=\frac{D_{r+1}(T)\left(\begin{array}{l}
\xi, \eta_{1}, \ldots, \eta_{r} \\
x, y_{1}, \ldots, y_{r}
\end{array}\right)}{D_{r}(T)\left(\begin{array}{l}
\eta_{1}, \ldots, \eta_{r} \\
y_{1}, \ldots, y_{r}
\end{array}\right)}
\end{aligned}
$$

The elements $\zeta_{1}, \ldots, \zeta_{r}$ are linearly independent in $\Xi$, and so are $z_{1}, \ldots, z_{r}$ in $X$. 
The equation

$$
x+T c=x_{0}
$$

has $a$ solution $x$ if and only of $\zeta_{i} x_{0}=0$ for $i=1, \ldots, r$. Then the general form of the solution is

$$
x=B x_{0}+c_{1} z_{1}+\ldots+c_{r} z_{r} .
$$

The adjoint equation

$$
\xi+\xi T=\xi_{0}
$$

has a solution $\xi$ if and only if $\xi_{0} z_{i}=0$ for $i=1, \ldots, r$. Then the general form of the solution is

$$
\xi=\xi_{0} B+c_{1} \zeta_{1}+\ldots+c_{r} \zeta_{r}
$$

In the case of $r=0$ Theorem (ii) asserts that

$$
\frac{D_{1}(T)}{D_{0}(T)}=(I+T)^{-1} \text {. }
$$

§ 5. Some identities for the Carleman determinant and subdeterminants. Now we shall prove that

(iii) $D_{0}(T)$ is the only analytic function on the Banach space $\mathcal{S}$ of all Hilbert-Schmidt operators, such that

$$
D_{0}^{\prime}\left(T ;(I+T) T_{1}\right)=-D_{0}(T) \cdot \operatorname{tr}\left(T T_{1}\right) \quad\left(T, T_{1} \in \widetilde{\Xi}\right)
$$

$$
D_{0}(0)=1
$$

First consider the case where $D_{0}(T) \neq 0$, i. e. $(I+T)^{-1}$ exists. Let

$$
S=(I+T)^{-1}-I \text {. }
$$

Since $S=-T-S T$ and $\mathcal{S}$ in an ideal in $\mathfrak{D}_{1}$, we have $S \epsilon \mathfrak{S}$. The identity (33) can be written

$$
D_{0}(T)(I+S)=D_{1}(T) \text {. }
$$

This equality and (19) imply

$$
D_{0}^{\prime}(T ; x \cdot \xi)=D_{0}(T) \cdot \xi S x .
$$

The number $\xi S x$ is the trace of the product of $S$ and the one-dimensional operator $T_{1}=\xi \cdot x$. Thus the last equality can be written

$$
D_{0}^{\prime}\left(T ; T_{1}\right)=D_{0}(T) \cdot \operatorname{tr} S T_{1}
$$

for all one-dimensional operators $T_{1}$. By linearity, (36) holds for all finitely dimensional operators $T_{1}$. By continuity, it holds for all $T_{1} \in \subseteq$ since the set of all finitely dimensional operators is dense in the Banach space $\mathfrak{S}$. Replacing in (36) $T_{1}$ by $(I+T) T_{1}$ we get (31) under the hypothesis that
$D_{0}(T) \neq 0$. Since $D_{0}(T)$ is analytic, the set $\left\{T: D_{0}(T)=0\right\}$ is nowhere dense in $\mathfrak{G}$. Hence it follows, by continuity, that (34) holds for every $T \in \mathcal{S}$. By the definition on p. $331, D_{0}(0)=1$. Thus the Carleman determinant is a solution of the differential equation (33) with the initial condition (34).

The proof that it is the only solution of (33) with the initial condition (34) is similar to the proof of the corresponding part of the analogous Theorem 6 in Sikorski [4] $\S 6$. The proof of uniqueness given there yields also the uniqueness of solution in a neighbourhood of $0 \in \mathbb{S}$.

(iv) For sufficiently small Hilbert-Schmidt operators $T$ (e. g. for $\|T\|<1)$,

$$
D_{0}(T)=\exp \operatorname{tr}(\log (I+T)-T)
$$

the logarithm being defined by the infinite series:

$$
\log (I+T)=\sum_{n=1}^{\infty} \frac{(-1)^{n-1}}{n} T^{n} .
$$

This follows from (iii) since the analytic function on the right side of (37) satisfies, in a neighbourhood of $0 € \mathfrak{S}$, the differential equation (33) with the initial condition (34).

For every Hilbert-Schmidt operator $T$, such that $(I+T)^{-1}$ exists, let $D_{0}(T)=1$ and, for $n>0$, let $\mathscr{D}_{n}(T)$ be the $2 n$-linear bounded functional defined by the equality

$$
D_{n}(T)\left(\begin{array}{l}
\xi_{1}, \ldots, \xi_{n} \\
x_{1}, \ldots, x_{n}
\end{array}\right)=\left|\begin{array}{c}
\xi_{1}(I+T)^{-1} x_{1}, \ldots, \xi_{1}(I+T)^{-1} x_{n} \\
\ldots \ldots . \ldots . . . . . . \\
\xi_{n}(I+T)^{-1} x_{1}, \ldots, \xi_{n}(I+T)^{-1} x_{n}
\end{array}\right| .
$$

Obviously, $\mathscr{D}_{n}(T)$ is an analytic mapping from the open dense set $\left\{T: D_{0}(T) \neq 0\right\} \subset \mathcal{S}$ into $\mathfrak{D}_{n}$. By a general theorem on determinant systems (see Sikorski [3], p. 183)

$$
D_{n}(T)=D_{0}(T) \cdot \mathcal{D}_{n}(T) \quad(n=0,1,2, \ldots) .
$$

For every nuclear operator $T$, let

$$
\boldsymbol{D}_{0}(T), \boldsymbol{D}_{1}(T), \boldsymbol{D}_{2}(T), \ldots
$$

be the Leżański determinant system for $A=I+T$ (for definition, see e. g. Sikorski [4], § 2). We have

$$
(40) \quad \boldsymbol{D}_{n}(T)=\boldsymbol{D}_{0}(T) \cdot \mathcal{D}_{n}(T) \quad(n=0,1,2, \ldots)
$$

by the same argument as in the case of (39). 
Every nuclear operator is a Hilbert-Schmidt operator, and the set of all nuclear operators is a dense subset of $\mathfrak{G}$. The nuclear norm $\||T|\|$ of a nuclear operator $T$ satisfies the inequality

$$
\|T\| \leqslant\|T\| \|
$$

(for the definition of the nuclear norm see e. g. Sikorski [2], Theorem V).

(v) For every nuclear operator $T$,

$$
D_{n}(T)=\boldsymbol{D}_{n}(T) \cdot \exp -\operatorname{tr} T \quad(n=0,1,2, \ldots)
$$

[1] $(86))$

If $\||T \||<1$, we have (Michel and Martin [1] p. 88; see also Sikorski

Hence, by (37) and (41)

$$
\boldsymbol{D}_{0}(T)=\exp \operatorname{tr} \log (I+T)
$$

for $\||T|\|<1$. Since the both sides of $(43)$ are analytic functions on the Banach space of all nuclear operators (with the norm ||$T|\||)$, the identity (43) holds for all nuclear $T$. It follows from (43), (39) and (40) that (42) holds for all nuclear $T$ such that $D_{0}(T) \neq 0$, and consequently, by analyticity, for all nuclear $T$.

To formulate the next theorem, it will be more suggestive to denote the determinant $D_{0}(T)$ of $I+T$ by $D(I+T)$.

(vi) For all Hilbert-Schmidt operators $T_{1}, T_{2}$,

$$
\text { (44) } \quad D\left(\left(I+T_{1}\right)\left(I+T_{2}\right)\right)=D\left(I+T_{1}\right) \cdot D\left(I+T_{2}\right) \cdot \operatorname{exptr} T_{1} T_{2} \text {. }
$$

For nuclear $T_{1}, T_{2}$ the identity (44) follows from (43) and the known identity (Michel and Martin [1], p. 89; see also Sikorski [1], Theorem 2 and $[4]$, Theorem 5)

$$
\boldsymbol{D}\left(\left(I+T_{1}\right)\left(I+T_{2}\right)\right)=\boldsymbol{D}\left(I+T_{1}\right) \cdot \boldsymbol{D}\left(I+T_{2}\right)
$$

where, similarly, $\boldsymbol{D}(I+T)=\boldsymbol{D}_{0}(T)$. By continuity, (44) holds everywhere in $\mathfrak{S}$.

It follows from (44) that, in the case where $X$ is finitely dimensional, $D(I+T)$ does not coincide, in general, with the algebraic determinant of $I+T$.

§6. The Carleman-Fredholm subdeterminants. For every $T \epsilon \Subset$, let $T_{n}^{* m}$ be the $2 n$-linear functional $\epsilon \mathcal{O}_{n}$ defined by the equality

$T_{n}^{* m}\left(\begin{array}{l}\xi_{1}, \ldots, \xi_{n} \\ x_{1}, \ldots, x_{n}\end{array}\right)=\sum\left|\begin{array}{l}\xi_{1} T^{i_{1}} x_{1}, \ldots, \xi_{1} T^{i_{1}} x_{n} \\ \ldots \ldots \ldots \ldots . . . \\ \xi_{n} T^{i_{n}} x_{1}, \ldots, \xi_{n} T^{i_{n}} x_{n}\end{array}\right|=\sum\left|\begin{array}{l}\xi_{1} T^{i_{1}} x_{1}, \ldots, \xi_{1} T^{i_{n}} x_{n} \\ \ldots \ldots \ldots \ldots \ldots \\ \xi_{n} T^{i_{1}} x_{1}, \ldots, \xi_{n} T^{i_{n}} x_{n}\end{array}\right|$ where the summation is extended over all sequences $i_{1}, \ldots, i_{n}$ of positive integers whose sum is equal to $m+n$. It follows from (45) and (24) that

$$
T_{n}^{* m}\left(\begin{array}{c}
\xi_{1}, \ldots, \xi_{n} \\
x_{1}, \ldots, x_{n}
\end{array}\right)=T_{n}^{m}\left(\begin{array}{c}
\xi_{1} T, \ldots, \xi_{n} T \\
x_{1}, \ldots, x_{n}
\end{array}\right)=T_{n}^{m}\left(\begin{array}{c}
\xi_{1}, \ldots, \xi_{n} \\
T x_{1}, \ldots, T x_{n}
\end{array}\right) .
$$

Let $D_{m, n}^{*}(T)$ be the $2 n$-linear functional $\epsilon \mathfrak{O}_{n}$ defined as follows:

$$
\left.D_{n, m}^{*}(T)=\mid \begin{array}{cccccccc}
T_{n}^{* 0}, & m, & 0, & 0, & 0, & \ldots, & 0, & 0 \\
T_{n}^{* 1}, & 0, & m-1, & 0, & 0, & \ldots, & 0, & 0 \\
T_{n}^{* 2}, & \operatorname{tr} T^{2}, & 0, & m-2, & 0, & \ldots, & 0, & 0 \\
T_{n}^{* 3}, & \operatorname{tr} T^{3}, & \operatorname{tr} T^{2}, & 0, & m-3, & \ldots, & 0, & 0 \\
\ldots & \ldots & \ldots & \ldots & \ldots & \ldots & \ldots & \ldots
\end{array}\right] .
$$

It follows from $(47),(46)$ and $(23)$ that

(48) $\quad D_{n, m}^{*}(T)\left(\begin{array}{c}\xi_{1}, \ldots, \xi_{n} \\ x_{1}, \ldots, x_{n}\end{array}\right)=D_{n, m}\left(\begin{array}{c}\xi_{1} T, \ldots, \xi_{n} T \\ x_{1}, \ldots, x_{n}\end{array}\right)=D_{n, m}\left(\begin{array}{c}\xi_{1}, \ldots, \xi_{n} \\ T x_{1}, \ldots, T x_{n}\end{array}\right)$.

Clearly $D_{n, m}^{*}(T)$ is a homogeneous polynomial of $T$ of the degree $n+m$ with values in $\mathfrak{D}_{n}$.

By (48) and (27) we have the following estimation of the norm of $D_{n, m}^{*}$ in $\mathfrak{O}_{n}$ :

(49) $\quad\left|D_{n, m}^{*}(T)\right| \leqslant 2^{n}(n+m)^{\frac{n+m}{2}}(2 \sqrt{e})^{n+m}\|T\|^{m+n}$.

In the case $n=1$ the identity (48) can be written in the form

$$
D_{1, m}^{*}(T)=T D_{1, m}(T)=D_{1, m}(T) T \text {. }
$$

Hence it follows that $D_{1, m}^{*}(T)$ is a Hilbert-Schmidt operator and, by $\left(4^{\prime \prime}\right)$,

$$
\left\|D_{1, m}^{*}(T)\right\| \leqslant 2(m+1)^{\frac{m+1}{2}}(2 \sqrt{e})^{m+1}\|T\|^{m+1} .
$$

The $2 n$-linear functional $\epsilon \mathfrak{O}_{n}$ :

$$
D_{n}^{*}(T)=\sum_{m=0}^{\infty} \frac{1}{m !} D_{n, m}^{*}(T) \quad(n=1,2, \ldots)
$$

is called the $n$-th Carleman-Fredholm subdeterminant of the operator $A=I+T$. It follows from (50) that the series (52) converges in norm in $\mathfrak{O}_{n}$. Moreover, in the case $n=1$, it follows from $(50)$ and $(51)$ that

$$
D_{1}^{*}(T)=T D_{1}(T)=D_{1}(T) T
$$


$D_{1}^{*}(T)$ is a Hilbert-Schmidt operator, and the series

$$
D_{1}^{*}(T)=\sum_{m=0}^{\infty} D_{1, m}^{*}(T)
$$

of Hilbert-Schmidt operators converges in the norm \|\| in $\mathfrak{S}$.

By (52), (48) and (21)

(55) $\quad D_{n}^{*}(T)\left(\begin{array}{l}\xi_{1}, \ldots, \xi_{n} \\ x_{1}, \ldots, x_{n}\end{array}\right)$

$$
=D_{n}(T)\left(\begin{array}{c}
\xi_{1} T, \ldots, \xi_{n} T \\
x_{1}, \ldots, x_{n}
\end{array}\right)=D_{n}(T)\left(\begin{array}{c}
\xi_{1}, \ldots, \xi_{n} \\
T x_{1}, \ldots, T x_{n}
\end{array}\right) .
$$

Let

$$
D_{0}^{*}(T)=D_{0}(T)
$$

The sequence

$$
D_{0}^{*}(T), D_{1}^{*}(T), D_{2}^{*}(T), \ldots
$$

is not a determinant system for the operator $A=I+T$ in the sense defined by Sikorski [3] since, instead of (31), (32), it satisfies the following identities:

$$
\begin{aligned}
D_{n+1}^{*}(T) & \left(\begin{array}{c}
\xi_{0} A, \xi_{1}, \ldots, \xi_{n} \\
x_{0}, x_{1}, \ldots, x_{n}
\end{array}\right) \\
& =\sum_{i=0}^{n}(-1)^{i} \xi_{0} T x_{i} \cdot D_{n}^{*}(T)\left(\begin{array}{c}
\xi_{0}, \ldots . . . . . ., \xi_{n} \\
x_{0}, \ldots, x_{i-1}, x_{i+1}, \ldots, x_{n}
\end{array}\right),
\end{aligned}
$$

$$
\begin{aligned}
D_{n+1}^{*}(T) & \left(\begin{array}{c}
\xi_{0}, \xi_{1}, \ldots, \xi_{n} \\
A x_{0}, x_{1}, \ldots, x_{n}
\end{array}\right) \\
& =\sum_{i=0}^{n}(-1)^{i} \xi_{i} T x_{0} \cdot D_{n}^{*}(T)\left(\begin{array}{c}
\xi_{0}, \ldots, \xi_{i-1}, \xi_{i+1}, \ldots, \xi_{n} \\
x_{1}, \ldots \ldots . . . \ldots, x_{n}
\end{array}\right) .
\end{aligned}
$$

However, it can be used to solve the equations $A x=x_{0}$ and $\xi A=\xi_{0}$. By the same method as in Sikorski [4] $\$ 3$ we can prove the following theorem:

(vii) Let $T$ be a Hilbert-Schmidt operator. The smallest integer $r$ such that $D_{r}^{*}(T) \neq 0 \in \mathfrak{D}_{r}$ is equal to the smallest integer $r$ such that $D_{r}(T) \neq 0 \in \mathfrak{O}_{r}$. Then $D_{r}^{*}(T)=(-1)^{r} D_{r}(T)$. Let $\eta_{1}, \ldots, \eta_{r}, y_{1}, \ldots, y_{r}$ be such that $D_{r}^{*}\left(\begin{array}{l}\dot{\eta}_{1}, \ldots, \dot{\eta}_{r} \\ y_{1}, \ldots, y_{r}\end{array}\right)$ $\neq 0$. Then there exist elements $\zeta_{1}^{*}, \ldots, \zeta_{r}^{*}, z_{1}^{*}, \ldots, z_{r}^{*}$ and a Hilbert-Schmidt operator $B^{*}$ such that for all $\xi, x$

$$
\begin{aligned}
\zeta_{i}^{*} x=\frac{D_{r}^{*}(T)\left(\begin{array}{l}
\eta_{1}, \ldots \ldots, \ldots, \ldots, \eta_{r} \\
y_{1}, \ldots, y_{i-1}, x, y_{i+1}, \ldots, y_{r}
\end{array}\right)}{D_{r}^{*}(T)\left(\begin{array}{l}
\eta_{1}, \ldots, \eta_{r} \\
y_{1}, \ldots, y_{r}
\end{array}\right)} \\
\xi z_{i}^{*}=\frac{D_{r}^{*}(T)\left(\begin{array}{l}
\eta_{1}, \ldots, \eta_{i-1}, \xi, \eta_{i+1}, \ldots, \eta_{r} \\
y_{1}, \ldots \ldots, \ldots, \ldots, y_{r}
\end{array}\right)}{D_{r}^{*}(T)\left(\begin{array}{l}
\eta_{1}, \ldots, \eta_{r} \\
y_{1}, \ldots, y_{r}
\end{array}\right)} \\
\xi B^{*} x=\frac{D_{r+1}^{*}(T)\left(\begin{array}{l}
\xi, \eta_{1}, \ldots, \eta_{r} \\
x, y_{1}, \ldots, y_{r}
\end{array}\right)}{D_{r}^{*}(T)\left(\begin{array}{l}
\eta_{1}, \ldots, \eta_{r} \\
y_{1}, \ldots, y_{r}
\end{array}\right)} .
\end{aligned}
$$

The elements $\zeta_{1}^{*}, \ldots, \zeta_{r}^{*}$ are linearly independent in $\Xi$, and so are $z_{1}^{*}, \ldots, z_{r}^{*}$ in $X$.

The equation

$$
x+T x=x_{0}
$$

has a solution $x$ if and only if $\zeta_{i}^{*} x_{0}=0$ for $i=1, \ldots, r$. Then the general form of the solution is

$$
x=x_{0}-B^{*} x_{0}+c_{1} z_{1}^{*}+\ldots+c_{r} z_{r}^{*} .
$$

The adjoint equation

$$
\xi+\xi T=\xi_{0}
$$

has a solution $\xi$ if and only if $\xi_{0} z_{i}^{*}=0$ for $i=1, \ldots, r$. Then the general form of the solution is

$$
\xi=\xi_{0}-\xi_{0} B^{*}+c_{1} \zeta_{1}^{*}+\ldots+c_{r} \zeta_{r}^{*} .
$$

In the case of $r=0$ Theorem (vii) asserts that

$$
I-\frac{D_{1}^{*}(T)}{D_{0}^{*}(T)}=(I+T)^{-1}
$$

\$ 7. An integral model. Let $\mu$ be a measure defined on a $\sigma$-field of subsets of a set $\Gamma$. The integrals taken over the whole space $\Gamma$ will be denoted, for brevity, by $\int f(t) d t$ instead of $\int_{T} f(t) d \mu(t)$, and similarly for multiple integrals.

Suppose now that $X=\Xi=L^{2}(\Gamma, \mu)$. The class $\subseteq$ of all HilbertSchmidt operators coincides (see e. g. Smithies [1]) with the class of all integral operators $T$

$$
T x(s)=\int \tau(s, t) x(t) d t
$$


where

(59)

$$
\|T\|=\sqrt{\iint|\tau(s, t)|^{2} d s d t}<\infty,
$$

i. e. $\tau \in \boldsymbol{L}^{2}(\Gamma \times \Gamma, \mu \times \mu)$.

Similarly as in Sikorski [4], §4, we can prove that

(60) $\quad D_{n, m}^{*}(T)\left(\begin{array}{l}\xi_{1}, \ldots, \xi_{n} \\ x_{1}, \ldots, x_{n}\end{array}\right)$

$=\int \ldots \int \vartheta_{n, m}^{*}\left(\begin{array}{c}s_{1}, \ldots, s_{n} \\ t_{1}, \ldots, t_{n}\end{array}\right) \xi_{1}\left(s_{1}\right) \cdot \ldots \cdot \xi_{n}\left(s_{n}\right) \cdot x_{1}\left(t_{1}\right) \cdot \ldots \cdot x_{n}\left(t_{n}\right) d s_{1} \ldots d s_{n} d t_{1} \ldots d t_{n}$,

where

(61) $\quad \vartheta_{n, m}^{*}\left(\begin{array}{c}s_{1}, \ldots, s_{n} \\ t_{1}, \ldots, t_{n}\end{array}\right)$

$$
\begin{aligned}
& T\left(s_{1}, t_{1}\right), \ldots, T\left(s_{1}, t_{n}\right), T\left(s_{1}, r_{1}\right), T\left(s_{1}, r_{2}\right) T,\left(s_{1}, r_{3}\right), \ldots, T\left(s_{1}, r_{m}\right)
\end{aligned}
$$

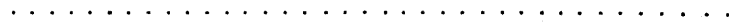

$$
\begin{aligned}
& T\left(s_{n}, t_{1}\right), \ldots, T\left(s_{n}, t_{n}\right), T\left(s_{n}, r_{1}\right), T\left(s_{n}, r_{2}\right), T\left(s_{n}, r_{3}\right), \ldots, T\left(s_{n}, r_{m}\right) \\
& =\int \ldots \int\left[\begin{array}{ccc}
T\left(r_{1}, t_{1}\right), \ldots, T\left(r_{1}, t_{n}\right), & 0, & T\left(r_{1}, r_{2}\right), T\left(r_{1}, r_{3}\right), \ldots, T\left(r_{1}, r_{m}\right) \\
T\left(r_{2}, t_{1}\right), \ldots, T\left(r_{2}, t_{n}\right), T\left(r_{2}, r_{1}\right), & 0, & T\left(r_{2}, r_{3}\right), \ldots, T\left(r_{2}, r_{m}\right)
\end{array} \mid \times\right. \\
& T\left(r_{3}, t_{1}\right), \ldots, T\left(r_{3}, t_{n}\right), T\left(r_{3}, r_{1}\right), T\left(r_{3}, r_{2}\right), \quad 0, \quad \ldots, T\left(r_{3}, r_{m}\right) \\
& T\left(r_{m}, t_{1}\right), \ldots, T\left(r_{m}, t_{n}\right), T\left(r_{m}, r_{1}\right), T\left(r_{m}, r_{2}\right), T\left(r_{m}, r_{3}\right) \ldots \ldots
\end{aligned}
$$

Thus formally

(62) $\quad D_{n}^{*}(T)\left(\begin{array}{l}\xi_{1}, \ldots, \xi_{n} \\ x_{1}, \ldots, x_{n}\end{array}\right)$

$$
=\int \ldots \int \vartheta_{n}^{*}\left(\begin{array}{c}
s_{1}, \ldots, s_{n} \\
t_{1}, \ldots, t_{n}
\end{array}\right) \xi_{1}\left(s_{1}\right) \ldots \xi_{n}\left(s_{n}\right) x_{1}\left(t_{1}\right) \ldots x_{n}\left(t_{n}\right) d s_{1} \ldots d s_{n} d t_{1} \ldots d t_{n}
$$

where

(63)

$$
\vartheta_{n}^{*}\left(\begin{array}{c}
s_{1}, \ldots, s_{n} \\
t_{1}, \ldots, t_{n}
\end{array}\right)=\sum_{m=0}^{\infty} \frac{1}{m !} \vartheta_{n, m}^{*}\left(\begin{array}{c}
s_{1}, \ldots, s_{n} \\
t_{1}, \ldots, t_{n}
\end{array}\right) .
$$

The problem under which conditions the series (63) converges, in some sense, to a function $\vartheta_{n}^{*}\left(\begin{array}{l}s_{1}, \ldots, s_{n} \\ t_{1}, \ldots, t_{n}\end{array}\right)$ and relation (62) holds, has not been investigated in detail. For $n=1$, it follows from $\S 6$ (54) that $\vartheta_{1, m}^{*} \in \boldsymbol{L}^{2}(\Gamma \times \Gamma, \mu \times \mu)$, the series

$$
\vartheta_{1}^{*}\left(\begin{array}{l}
s \\
t
\end{array}\right)=\sum_{m=0}^{\infty} \vartheta_{1, m}^{*}\left(\begin{array}{l}
s \\
t
\end{array}\right)
$$

converges in $\boldsymbol{L}^{2}(\Gamma \times \Gamma, \mu \times \mu)$ and the function $\vartheta_{1}^{*} \in \boldsymbol{L}^{2}(\Gamma \times \Gamma, \mu \times \mu)$ satisfies the identity:

$$
D_{1}^{*}(T)\left(\begin{array}{l}
\xi \\
x
\end{array}\right)=\iint \vartheta_{1}^{*}\left(\begin{array}{l}
s \\
t
\end{array}\right) \xi(s) x(t) d s d t
$$

If every one-point subset of $\Gamma$ has a positive measure, then the series (63) converges pointwise and the identity (62) holds $(n=1,2, \ldots)$.

To give an integral formula for $D_{n}(T)$ we have to introduce, simil ærly as in Sikorski [4], $\$ 4$, a substitute of the Dirac delta distribution, i. e. a formal expression $\delta(s, t)$ which is a formal kernel of the operator $I$. By definition,

$$
\begin{gathered}
\int \delta(s, t) x(t) d t=x(s), \quad \int \xi(s) \delta(s, t) d s=\xi(t), \\
\iiint \delta(s, t) \tau_{1}(t, r) \tau_{2}(r, s) d r d s d t=\iint \tau_{1}(t, s) \tau(s, t) d s d t,
\end{gathered}
$$

etc. (for details, see Sikorski [4], §4). Then we can write formally

$$
\text { (64) } \begin{aligned}
& D_{n, m}(T)\left(\begin{array}{c}
\xi_{1}, \ldots, \xi_{n} \\
x_{1}, \ldots, x_{n}
\end{array}\right) \\
= & \int \ldots \int \vartheta_{n, m}\left(\begin{array}{c}
s_{1}, \ldots, s_{n} \\
t_{1}, \ldots, t_{n}
\end{array}\right) \xi_{1}\left(s_{1}\right) \ldots \xi_{n}\left(s_{n}\right) x_{1}\left(t_{1}\right) \ldots x_{n 2}\left(t_{n}\right) d s_{1} \ldots d s_{n} d t_{1} \ldots d t_{n},
\end{aligned}
$$

where

(6丂) $\quad \vartheta_{n, m}\left(\begin{array}{l}s_{1}, \ldots, s_{n} \\ t_{1}, \ldots, t_{n}\end{array}\right)$

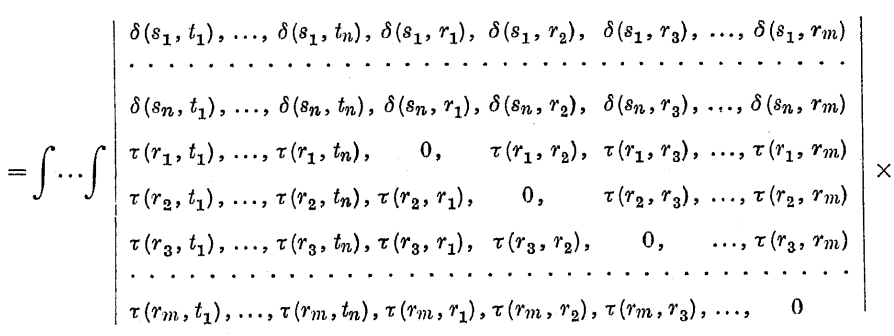

and

$$
\text { (66) } \begin{aligned}
& D_{n}(T)\left(\begin{array}{l}
\xi_{1}, \ldots, \xi_{n} \\
x_{1}, \ldots, x_{n}
\end{array}\right) \\
= & \int \ldots \int \vartheta_{n}\left(\begin{array}{c}
s_{1}, \ldots, s_{n} \\
t_{1}, \ldots, t_{n}
\end{array}\right) \xi_{1}\left(s_{1}\right) \ldots \xi_{n}\left(s_{n}\right) x_{1}\left(t_{1}\right) \ldots x_{n}\left(t_{n}\right) d s_{1} \ldots d s_{n} d t_{1} \ldots d t_{n}
\end{aligned}
$$


where

$$
\vartheta_{n}\left(\begin{array}{l}
s_{1}, \ldots, s_{n} \\
t_{1}, \ldots, t_{n}
\end{array}\right)=\sum_{m=0}^{\infty} \frac{1}{m !} \vartheta_{n, m}\left(\begin{array}{l}
s_{1}, \ldots, s_{n} \\
t_{1}, \ldots, t_{n}
\end{array}\right) .
$$

If every one-point subset of $\Gamma$ has a positive measure, then $\delta(s, t)$ and consequently, also $\vartheta_{n, m}\left(\begin{array}{c}s_{1}, \ldots, s_{n} \\ t_{1}, \ldots, t_{n}\end{array}\right), \vartheta_{n}\left(\begin{array}{l}s_{1}, \ldots, s_{n} \\ t_{1}, \ldots, t_{n}\end{array}\right)$ are functions, and the series (66) converges pointwise $(n=1,2, \ldots)$.

\section{References}

T. Carleman [1] Zur Theorie der linearen Integralgleichungen, Math. Zeitschrift 9 (1921), p. $196-217$.

I. Fredholm [1] Sur une classe d'équations fonctionelles, Acta Math. 27 (1903), p. $365-390$

A. Grothendieck [1] La théorie de Fredholm, Bull. Soc. Math. France 84 (1956), p. 319-384.

- [2] Produits tensoriels topologique et espaces nucléaires, Memoires Amer. Math. Soc. 1955

B. Fuglede and R. V. Kadison [1] On determinants and a property of trace in finite factors, Proc. Nat. Acad. Sci. USA 37 (1951), p. 425-431.

- [2] Determinant theory in finite factors, Annals of Math. 55 (1952), p. $520-530$

D. Hilbert [1] Grundzüge einer allgemeinen Theorie der linearen Gleichungen, Leipzig 1912.

E. Hille and R.S. Phillips [1] Functional analysis and semi-groups, Providence 1957.

E. Hille and J. D. Tamarkin [1] On the eharacteristic values of linear integral equations, Proc. Nat. Acad. Sci. 14 (1928), p. $911-914$.

- [2] On the characteristic values of linear integral equations, Acta Math. 57 (1931), p. $1-76$.

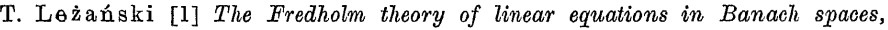
Studia Math. 13 (1953), p. 244-276.

- [2] Sur les fonetionelles multiplicatives, Studia Math. 14 (1953), p. 13-23.

A. D. Michel and R. S. Martin [1] Some expansions in vector space, Journ. Math. Pures et Appl. 13 (1934), p. 69-91.

A. F. Ruston [1] On the Fredholm theory of integral equations for operators belonging to the trace class of a general Banach space, Proc. London Math. Soc. (2) 53 (1951), p. $109 \cdot 124$.

- [2] Direct products of Banach spaces and linear functional equations, Proc. London Math. Soc. (2) 1 (1951), p. 327-384.

R. Sikorski [1] On Leżański's determinants of linear equations in Banach spaces, Studia Math. 14 (1953), p. 24-48.

- [2] On determinants of Leżaństi and Ruston, ibidem 16 (1957), p. 99-112.

- [3] Determinant systems, ibidem 18 (1959), p. 161-186.

- [4] Remarks on Leíański's determinants, ibidem 20 (1961), p. 145-161.

- [5] The determinant theory in Banach spaces, Coll. Math. 8 (1961), p. 141-198.

F. Smithies [1] The Fredholm theory of integral equations, Duke Math. Journ. 8 (1941), p. $107-130$.

- [2] Integral equations, Cambridge 1958.

Reşu par la Rédaction le 8. 10. 1960

\section{Réfractions non-hilbertiennes \\ d'une transformation symétrique bornée}

par

G. L. KRABBE (Lafayette, Ind.)

1. Introduction. Dans tout ce qui suit, $\mu$ est une mesure sur un clan de parties d'un ensemble $\omega$, et $L^{0}$ est l'ensemble des fonctions étagées $\mu$-mesurables sur $\omega$. Remarquons que $L^{0}$ est partout dense dans l'espace de Banach $\mathfrak{X}_{r}=\left\{x:\|x\|_{1 / r}<_{1}<\infty\right\}$, où

$$
\|x\|_{1 / r}=\left(\int_{\infty}|x|^{1 / r} d \mu\right)^{r}
$$

nous supposerons désormais que $0<r<1$. Soit $\mathcal{H}$ l'ensemble des transformations symétriques bornées de l'espace hilbertien $\mathfrak{X}_{1 / 2}$. Posons $H \epsilon \mathcal{H}$ et

$$
|H|_{r}=\sup \left\{\|H x\|_{1 / r}: x \in L^{0} \text { et }\|x\|_{1 / r} \leqslant 1\right\} .
$$

Soit $H^{0}$ la restriction de $H$ à $L^{0}$, et désignons par $H_{r}$ le prolongement linéaire continu de $H^{0}$ à $\mathfrak{X}_{r}$; nous dirons que $H_{r}$ est la "réfraction" de $H$. Précisons: $H_{r}$ est l'unique prolongement de $H^{0}$ appartenant à l'ensemble $\varepsilon_{r}$ des endomorphismes continus de $\mathfrak{X}_{r}$. Il est clair que $H$ possèdera une réfraction si et seulement si $|H|_{r} \neq \infty$.

Rappelons qu'il exist une bijection $H \rightarrow E^{H}$ de $\Re$ sur l'ensemble des familles spectrales (cf. [13], pp. 174-176). Nous noterons $\mathcal{H}_{i}$ l'ensemble des transformations $H$ appartenant à $\mathcal{H}$ telles que

$$
\infty \neq \sup _{\infty<\lambda<\infty}\left|E^{H}(\lambda)\right|_{s} \quad \text { lorsque } \quad 0<s<1 .
$$

Nous supposerons désormais que $H \epsilon \mathscr{H}_{i}$. On verra (au no. 2.8) que $H$ possède une réfraction $H_{r}$; en outre, $H_{r}$ admet une décomposition spectrale (bien que $H_{r}$ ne soit pas nécessairement un ,spectral operator" dans le sens de $N$. Dunford: voir no. 3.4 et $\S 5$ ). La réfraction hérite de $H$ d'autres propriétés spectrales $\left(^{1}\right)$. Par exemple, $H_{r}$ possède un ,calcul

(1) Il serait intéressant de déterminer quelles autres propriétés spectrales de $H$ sont heritées par $H_{r}$. A ce propos, voir $\S 5$. 